\title{
Research on an Iot Based Air Pollution Monitoring System
}

\author{
Vijayakumar Sajjan, Pramod Sharma
}

\begin{abstract}
Humankind, moving to a period centered upon improvement has overlooked the significance of supportability and has been the real guilty party behind the rising Pollution levels in the world's air among all other living life forms. The Pollution levels at certain spots have come to such high degrees that they have begun hurting our very own It will being. An IoT based Air Pollution observing framework incorporates a MQ Series sensor interfaced to a Node MCU outfitted with an ESP8266 WLAN connector to send the sensor perusing to a Thing Speak cloud. Further extent of this work incorporates an appropriate AI model to foresee the air Pollution level and an anticipating model, which is fundamentally a subset of prescient displaying. As age of poisonous gases from ventures, vehicles and different sources is immensely expanding step by step, it winds up hard to control the dangerous gases from dirtying the unadulterated air. In this paper a practical air Pollution observing framework is proposed. This framework can be utilized for observing Pollutions in demeanor of specific territory and to discover the air peculiarity or property examination. The obligated framework will concentrate on the checking of air poisons concentrate with the assistance of mix of Internet of things with wireless sensor systems. The investigation of air quality should be possible by figuring air quality index (AQI).
\end{abstract}

\section{Keywords: MCU, WLAN, AQI Etc.}

\section{INTRODUCTION}

Air contamination can be characterized as nearness of moment particulars that bothers the working of common procedures and furthermore creates unfortunate wellbeing impacts. In another way contamination can influence the characteristic periodicity and furthermore can irritate the wellbeing of person. As modernization and automation is becoming in all respects widely Pollution is likewise getting presented everywhere way.

It has been seen that in mechanically creating or created nations human wellbeing get significantly influenced due to Air Pollution, where there is no framework to screen it or monitor it [1]. In late explores it has been demonstrated that there is a high connection batten's climatic toxins and maladies like asthma and lung related ailments. Air Pollution is currently a noteworthy worry over the globe and WHO has built up specific rules to confine the cutoff points of specific gases like O3, NO2, SO2 [2]. The Air Quality Index estimation and Pollution observing are mostly done AQM stations that are essentially exact and precise. They show ideal unwavering quality and viable in estimating a wide scope of air toxins. Be that as it may, even after every one of these stations slack fundamentally in three territories:

Revised Manuscript Received on July 18, 2019. University Of Technology, Jaipur, Rajasthan, India.(Email: vijay.sajjan26@gmail.com)

Pramod Sharma, Professor In ECE Dept, University Of Technology, Jaipur, Rajasthan, India.
Vijayakumar Sajjan, Research scholar, Department of ECE,

1) Infrastructure, essential for establishment as a result of the colossal size, 2) Operational necessities are basically mind boggling, 3) The common costs of setting up, day by day support and alignment.

Thinking about the evil impacts of Pollution on people, in 2012, one out of eight of all out worldwide passings were brought about via air Pollution which was 7 million unexpected losses all around [3]. These passings were a consequence of various ailments, for example, ischemic coronary illness, interminable obstructive pneumonic sickness, stroke, lung malignant growth and intense lower respiratory diseases in youngsters [3]. The foundations for every one of those illnesses were related with outside and indoor air Pollution consolidated. Presently, in the event that one discussions about water Pollution, expending defiled water can prompt genuine medical problems in individuals and one may get influenced by hazardous waterborne ailments brought about by proto zoans, infections and microscopic organisms', for example, amoebiasis, hepatitis An, E coli and loose bowels. According to the WHO (world health association) these sicknesses have a portion of around $3.6 \%$ in the complete every day worldwide It right of ailments [4], and cause about 1.5 million human passings yearly. Additionally, commotion Pollution is likewise as unsafe as the other two sorts of Pollution as it might prompt hearing It takes, hypertension, ischemic coronary illness, irritation, and rest aggravation [5].

The system of ceaseless internet checking utilizes sensors to screen the numerical value, and afterward transfer to control focus by system. The method for information move incorporates wired and wireless frameworks. Despite the fact that framework is solid it is having weaknesses everywhere and dynamic range, for example, complex system cabling, costly and so forth. With widely creating correspondence advances, presently multi day's air Pollution checking framework is frequently planned in wireless mode. In any case, these modes are surprising expense in both establishment and support. Yet, on different handy cellular sensor systems have been quickly created during late decades and utilized on the huge systems at military, enterprises as well. In view of these focal points, it is currently being connected in ecological observing [4].In request to actualize such framework single integratedchip micro-controller alongside exhibit of sensors, IOT device and GPS-device is utilized. This framework estimates grouping of gases, for example, $\mathrm{CO}, \mathrm{NO} 2$ andSO2 utilizing electricity produced by chemical changes sensors. The equipment block accumulates air poisons levels likewise 
group them into the edge with GPS physical area, time and date.

To screen air quality, wireless sensor networks (WSNs) may be an incredible apparatus, since they can consequently gather air quality information. It will likewise assist us with keeping a working staff away from risk and a high security can be accomplish and it will likewise help the Government specialists to screen the air Pollution. The propose framework will concentrate on the observing of air poisons concentrate with the assistance of blend of Internet of things with wireless sensor systems. The investigation of air quality should be possible by figuring air quality record. This data will be shown on the website page by means of web continuously. Likewise the framework is practical which make its establishment conceivable in different territories.

\section{LITERATURE REVIEW}

1. Zigbee Based Wireless Air Pollution Monitoring System Using Low Costand Energy Efficient Sensors.

Mr.Vasim K. Ustad, Prof.A.S.Mali , Mr.SuhasS.Kibile, PG Student, Department of Electronics Engineering, Tatyasaheb Kore Institute of Engineering \& Technology, Warananagar, Maharashtra, India.

Air pollution is not only natural medical matters impact on creating nations alike. The strong effect of air pollution on wellbeing are extremely mind blowing as there are a broad area of sources and their particular influence differ from one another. The synthetic substances reason an assortment of mankind and natural medical issues enlarge in air contamination impacts on condition also on human wellbeing. To screen this contamination WSN framework is expressed. The proposed framework comprises of a Unit of Mobile-DAQ and a fixed Internet-Enabled contamination observation System. The Mobile-DAQ unit incorporates a solitary chip microcontroller, air pollution sensors exhibit, and GPS Device. The Pollution-Server is a top of the line individual computer application server with Internet network. The Mobile-DAQ unit assembles air toxins levels ( $\mathrm{CO}, \mathrm{NO} 2$, andSO2), and packs them in a casing with the GPS physic distribution, time, and date. The reason is to send the Pollution-Server by means of zig bee device. The pivotal-Server is interact to Google Maps to show the area of equipment. It can associate database server to the Pollution-Server for putting away the toxins range for future utilization by different user, for example, condition security offices, vehicles registration experts, and vacationer and insurance agencies.

\section{Pollution Monitoring System utilizing WSN in Visakhapatnam}

P.VijnathaRaju, M.Tech Student R.V.R.S.Aravind, Associate Professor Nova school of Engineering and TechnologyJangareddigudem, W.G Distict, AP, India, Department of ECE ,Sanketika Institute of Technology and Management, Visakhap'antnam, India.

As the technology increases, the level of robotic work (cutting the labor) in the practically all parts are likewise increments. WSN are grabing up the ground in all divisions of life; from homes to industrial facilities, from traffic control to natural checking. The air pollution monitoring system contains sensors to screen the intrigued pollution parameter with regards to condition. It reenacted the three air contaminations gases including $\mathrm{CO}, \mathrm{CO}_{2}$ and $\mathrm{SO}_{2}$ in air in light of the fact that these gases chooses the level of pollution. It can additionally apply the methodology in dissimilar house hold activities like spilling culinary gas in our homes, to caution the laborers in oil and gas sedulity to recognize the spillage and so on. This repetition makes the awareness in individuals in urban communities.

3. Wsn Based On Air Contamination Monitoring System In Metropolitan Cities

The WSN based air contamination observing framework [3] depends on AVR ATmega-32 Microcontroller. The sensor network is utilized to distinguish the sensor esteems from various detectors as MQ5, MQ7, temperature and dampness particularity. ID3 calculation is utilized to ascertain the qualities contemptible on likelihood. Bluetooth device is utilized to interface the control with customer and the customer associates with the server by means of web administrations. This framework not just computes the pollution present noticeable all around yet in addition can make a figure to stay away from future contamination in the specific dirtied zone. Here they consider essentially the substance Industry close Pune and the I.T. zone like Hinjewadi.

\section{IOT DEVICE PROTOTYPE}

IoT for the most part manages associating shrewd gadgets (implanted hardware gadgets) to Itb by tackling the upside of OSI layered Architecture. With regards to this work It propose a group of Air Quality Monitoring Sensor bits, which are utilized to quantify the convergence of Air contaminations noticeable all around. All the Air Sensors are interfaces with a minor implanted stage outfitted with system availability and are interconnected to Itb making it a worldwide system of associated things. It have essentially utilized the Node MCU which is an open source advancement sheets with ESP8266-12E chips. MQ-2 Gas Sensor is utilized to gather gas focus estimations. This sensor information would be caught and sent to the Thing Speak cloud for IoT based information procurement. Fig.1 demonstrates the proposed framework design. 


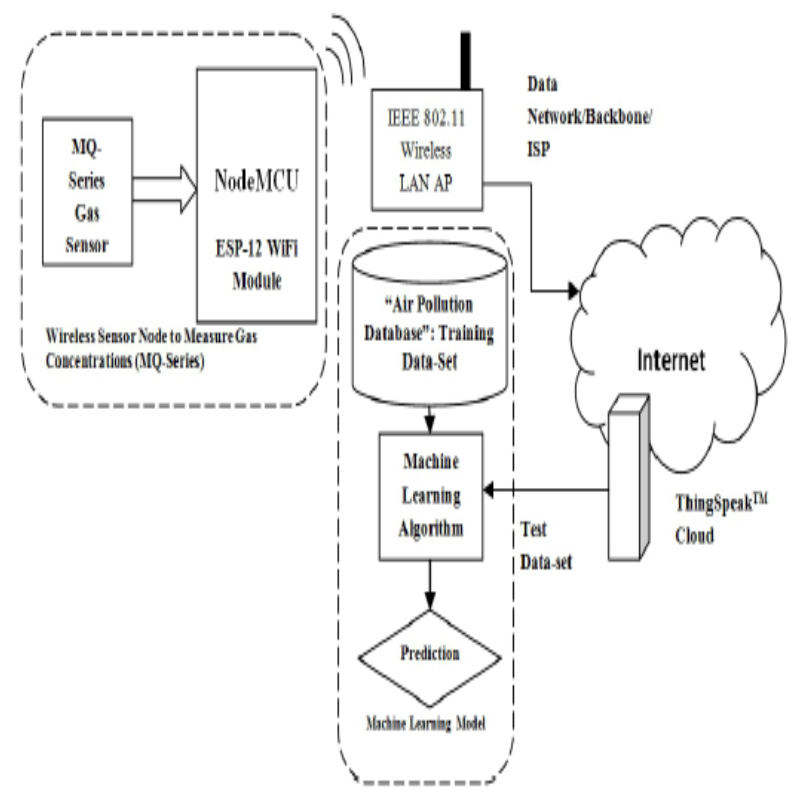

Fig. 1: Block diagram of proposed IoT based Air Pollution Monitoring System

Fig. 2 demonstrates the stick design of Node MCU Board which is utilized to interface the MQ2 gas sensor, ESP8266 WLAN connector is implanted inside the gadget.

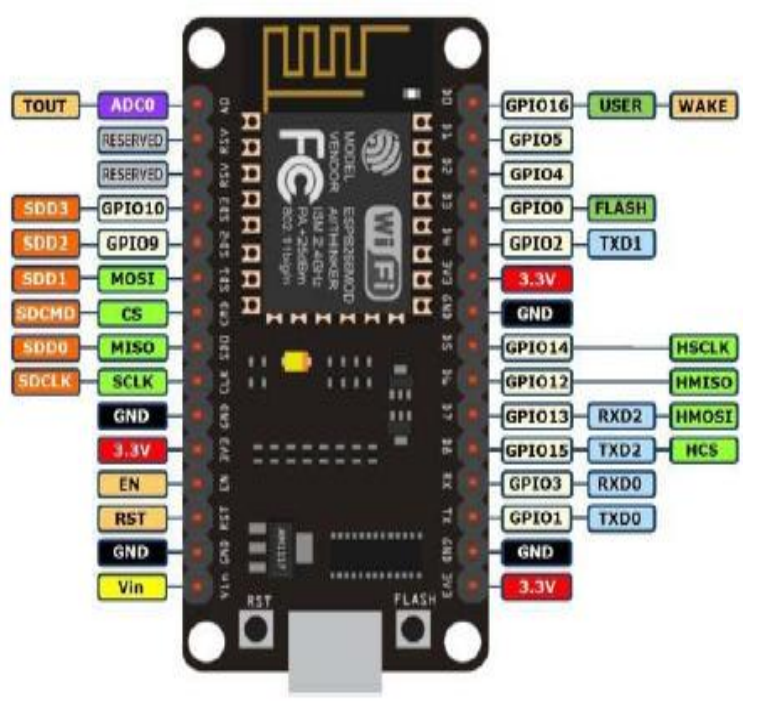

Fig. 2: Node MCU Board with PIN configuration

Air contamination observation module in Solapur City using WS

T.H.Mujawar,V.D.Bachuwar,S.S.Suryavanshi,

Ph.D

Department of Electronics Solapur University Solapur, Maharashtra, India.

Because of recent innovation there is pattern in scaling down of gadgets which requests to grow minimal effort sensor, low power and rough gadgets .In perspective on this WSN have picked up significance in different applications like Business, Agricultural, Domestic, Industries, Traffic control, and natural checking. The module contains Wireless detector arrange framework utilize to screen and control the air quality in Solapur city. Natural air contamination observing framework that measure, Suspended Particulate Matter, NOx, and SO2 are suggest. The conventional air quality checking framework, constrained by the Pollution regulator organization is amazingly costly. Investigative estimating gear is expensive, time and power devouring, and would seldom be able to be utilized for air quality announcing progressively. Endeavor has been made to create checking framework utilizing monetarily accessible standard poison gas sensors and CC2530ZDK board that utilizations $2.4 \mathrm{GHz}$ IEEE 802.15.4 standard, superior low power 8051 center, which will fill in as a hub in a Wireless Sensor Network. A particular docket made with Lab VIEW is made to design and direct the activity and the detecting estimations on the system utilized.

Electronic air contamination observing framework using smart telephon

ShilpaR. Khodve, A .N. kulkarni, dept. Of gadgets and media transmission, enthusiasm school of building and research, pune, india.

This framework have ARM7 LPC2138 is heart of the framework. Sensors like temperature, smoke, co, no are interfaced with microcontroller which is utilize the ecological checking air contamination and hand-off for burden like fan and LED. The transfer works on $12 \mathrm{v}$ power supply so It are giving this 12 volt to hand-off from optional side of transformer. The framework planned $5 \mathrm{v}$ power supply for the device LCD, MAX232, detectors which require 5v. In any case, controller LPC2138 and Bluetooth device require $+3.3 \mathrm{v}$ that It are giving by utilizing LM1117 IC that is in SMD bundle. The controller constantly peruses the estimation of detectors and presentations it on LCD, and by utilizing Bluetooth device these estimations of detectors are sent to android versatile. At the versatile side android server is intended to demonstrate the parameters around the world. These qualities are shown on the versatile window just as overall portable or PC. Information will be seen by the any designer from any apocalypse by this framework. On the off chance that whenever estimation of temperature sensor will increment by limit that It have set 40, FAN will on that is associated as a heap to hand-off. It implies if temperature builds transfer will on and it will on the fan. Essentially if estimation of smoke sensor will cross the limit esteem that is 45 , transfer will on the LED that is visual sign. What's more, again this expanded worth will likewise send to portable utilizing Bluetooth device. Various sensors are associated with ARM7 by means of inbuilt ADC. The yield of sensors having the data of various numerical values. ARM7 is associated Bluetooth device through Max232.The Bluetooth device is utilized to transferred the information to the Android telephone from Hardware. Android based server \& PC are interfaced with the same system by means of same Wi-Fi or keeping hotspot on of advanced mobile phone. A similar screen as work station is shown on the advanced mobile phone so the individual from Wireless area also see the present status of different parameters .It will initially interface the Android telephone \& PC. When the association is established the manager will probably screen remote sensor parameter utilizing the Android telephone. Additionally the a user can transferred data by means of his advanced mobile phone straight forwardly to computer. 


\section{PREDICTIVE AND FORECASTING MODELING}

ML chiefly manages computational techniques that improve the execution of mechanizing the verifying of gaining from experience [16]. The way toward taking in by a machine from complex arrangement of information and taking care of basic issues, being increasingly shrewd is the thing that AI is about. Much the same as, there's a standard climate gauging accomplished for the following day, similarly the contamination anticipating model can be utilized with the goal that individuals can take prudent steps.

It mean to precisely foresee centralizations of $\mathrm{O} 3, \mathrm{SO} 2$, $\mathrm{NO} 2$ and CO. For testing our model It have utilized an approved contamination dataset given by US EPA, which comprises of contamination information for 46 conditions of the USA over the most recent 15 years. The way toward structure the forecast models for the most part manage 3 stages:

1. Data Pre-preparing: The initial step of structure an expectation model is information pre-handling where information is cleaned, missing qualities are filled, anomalies are evacuated and furthermore information is masterminded in a manner to fit for the Machine Learning calculations.

2. Feature Engineering: Features are one of the main considerations which increment the forecast exactness such, day, month, time of the da $\mathrm{y}$, and so forth.

3. Building Forecasting Model: Model is worked to anticipate the future, for example on the inconspicuous information dependent on the verifiable information.

In preparing information know target factors are put away, highlights are chosen and these highlights are utilized in every one of the calculations. For the testing part the created models are cross-approved and assessed. The model was assessed utilizing cross validation methods dependent on Root mean square error (RMSE) and the mean absolute error (MAE). The models are actualized and their individual execution is assessed in this work, which gives a subjective proportion of model's exhibition. Mean Absolute Error (MAE) is the estimation of the normal greatness of the blunders in a lot of forecasts is determined without thinking about their course. MAE is the normal of total contrast of the whole test between the genuine perception and the expectation informational index, here all individual distinction have equivalent load as given in condition (1).

$$
M A E=\frac{1}{n} \sum_{j=1}^{n}\left|y_{j}-\hat{y}_{j}\right|
$$

For actualizing the model, all things considered, the best highlights and forecast model will be utilized for the concealed information. In the unpredictable natural changes the repetition of preparing and testing and sending will be done intermittently. This is an iterative procedure which ought to be done to improve the model execution. There are numerable calculations that can be executed, yet out of which Random Forest and XGBoost demonstrated to be the best models. XGBoost is another approach to state
"Outrageous Gradient Boosting". The articulation "Tendency Boosting" is proposed as a Greedy Function Approximation: A Gradient Boosting Machine, by Friedman. Slope boosting is presently one of the most Itll known strategies for proficient displaying of unthinkable datasets all things considered. XGBoost is a quick, versatile execution of angle boosting. XGBoost relies upon this special model. XGBoost is a usage of slope supported choice trees intended for speed and execution. The Gradient Boosting Model (GBM) in light of choice tree is a famous AI strategy. Slope boosting is an AI (ML) strategy normally utilized for relapse and grouping issues, which delivers an expectation model as a gathering of frail forecast models, in light of a choice trees. A phase astute design model is worked by GBM fundamentally the same as other boosting strategies. A self-assertive differentiable misfortune capacity is utilized to further sum them up by permitting streamlining.

A Autoregressive integrated moving normal (ARIMA) is one of the outstanding straight models in time plan deciding in the midst of the past three decades. Late research practices in foreseeing with artificial neural networks (ANNs) prescribe that ANNs can be a promising differentiating choice to the standard straight strategies. ARIMA models and ANNs are consistently differentiated and mixed ends with respect to the predominance in guaging models. Fig. 3 demonstrates the proposed framework design, which translates the preparation and testing informational index. The proposed design incorporates the preprocessing and highlight extraction with cross approval procedures.

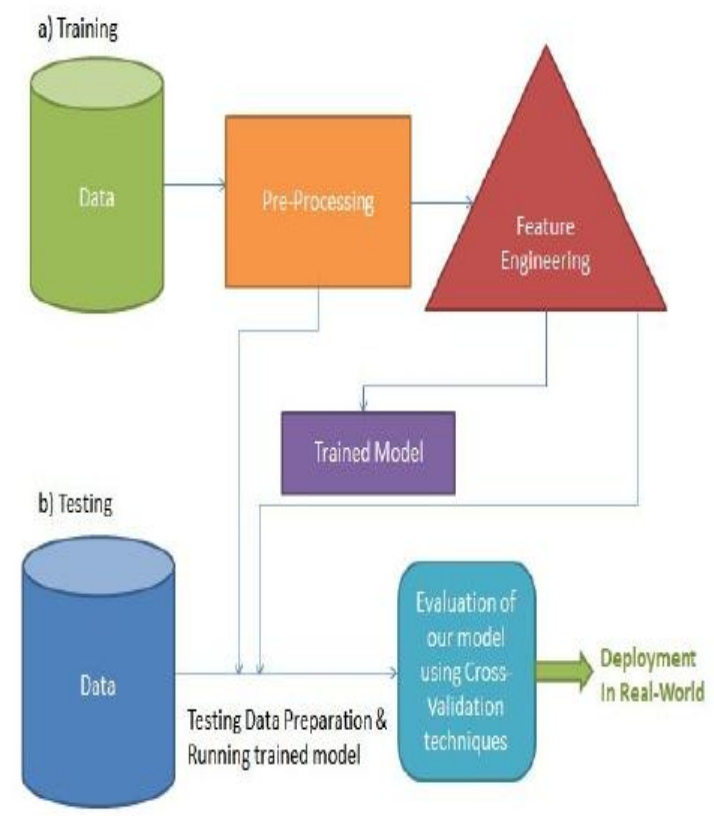

Fig. 3: Solution Architecture to build Predictive Model 


\section{RESULT ANALYSIS}

\section{Air pollution results}

Figure-4 demonstrates the raspberry pi terminal when it is observing air and commotion contamination levels. At the point when the air sensor esteem (set apart in red, $502 \mathrm{ppm}$ ) surpasses the limit ( $300 \mathrm{ppm})$, one can see that the notice is sent and affirmation is gotten by the raspberry pi in the following line. Likewise, it tends to be seen that when the air sensor esteem (set apart in blue) was underneath the limit, no notice was sent.

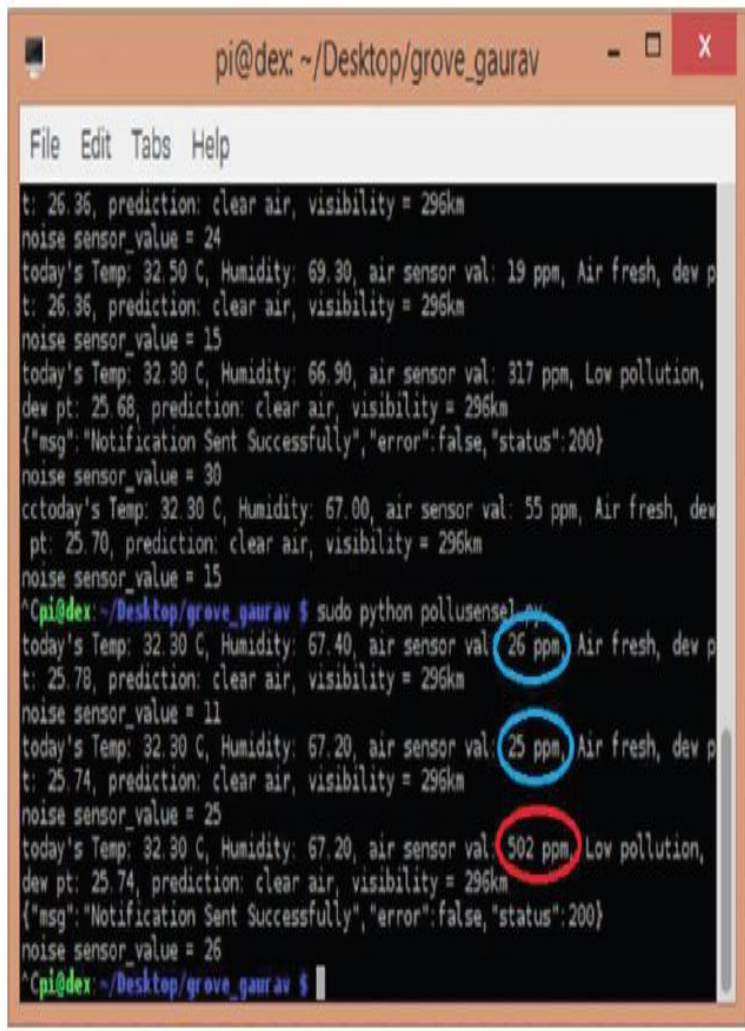

Fig. 4: Raspberry Pi terminal to show presence of air pollution with $300 \mathrm{ppm}$ as threshold.

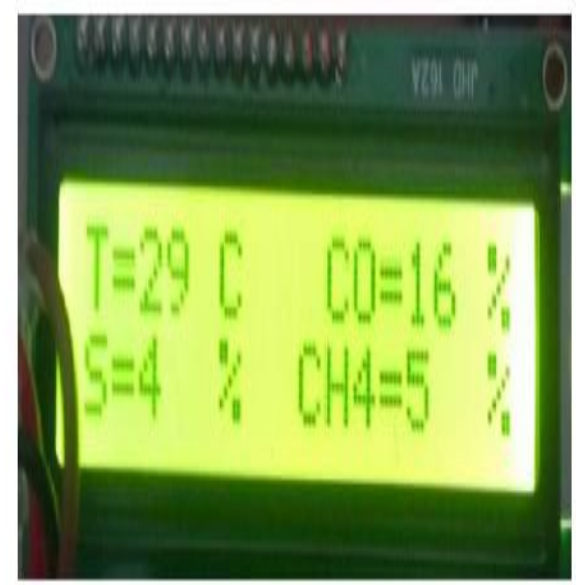

Fig.5 Parameter Display on LCD Screen
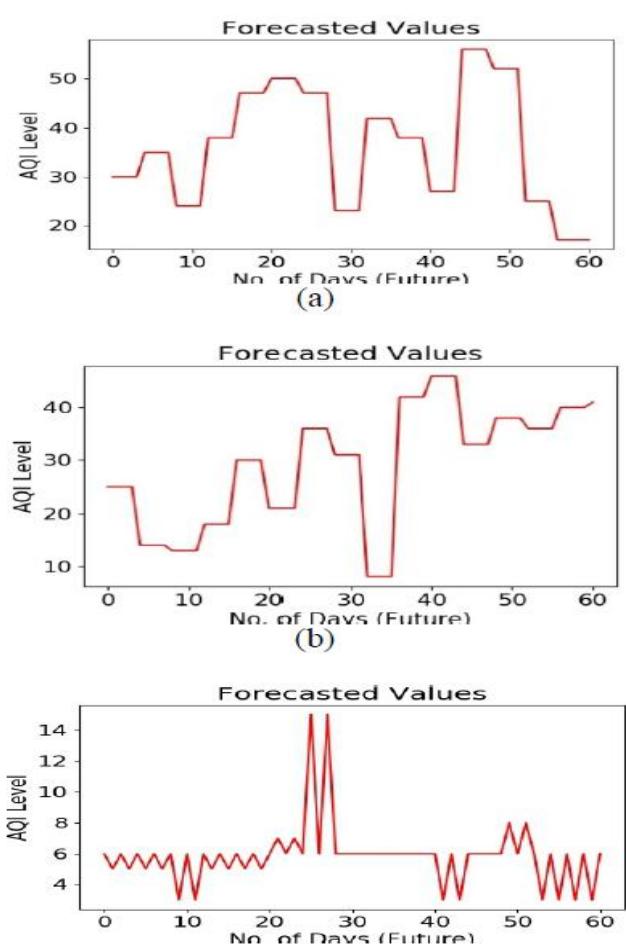

(c)

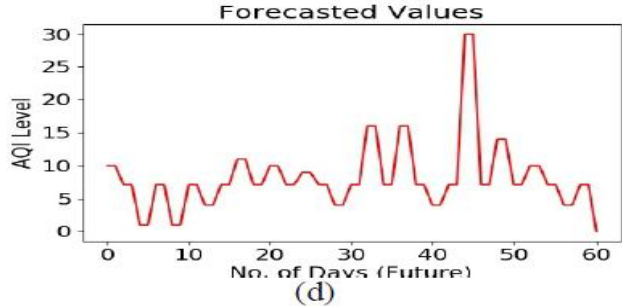

Fig. 6: ARIMA Time Series Future Forecasting: (a) $\mathrm{NO2}$ (b) $\mathrm{O3}$ (c) $\mathrm{CO}$ (d) $\mathrm{SO} 2$

\section{CONCLUSION}

Air quality is a basic issue that clearly impacts human prosperity. Air quality data are accumulated remotely from checking bits that are furnished with an assortment of vaporous additionally, meteorological sensors. This data are researched and used as a piece of envisioning obsession estimations of pollutions using keen machine to machine organize. Ongoing air contamination remote checking framework utilizes xbee though zigbee and gps based air contamination observing framework and wsn based air contamination checking framework utilizes zigbee for sending gathered poison data to the pc. In wireless sensor system based contamination checking framework a bluetooth system is utilized just as investigation is finished utilizing id3 calculation. Arduair air quality checking framework utilizes usb link for information move additionally ongoing chart plotting has done. All the above framework has short range to send information to the pc. Constant remote air contamination checking framework has utilized multi jump organize hoitver it is costlier due to libelium waps. 


\section{REFERENCES}

1. D.Yaswanth, Dr Syed Umar,-" A Study on Pollution Monitoring system inWireless Sensor Networks",D.Yaswanth et al | IJCSET |September 2013 | Vol 3, Issue 9, 324-328.

2. Anil H. Sonune, S.M.Hambarde,-" Monitoring and Controlling of Air Pollution Using Intelligent Control System",- International Journal of Scientific Engineering and Technology ISSN: 2277-1581,Volume No.4 Issue No5, pp: 310-313.

3. Martinez, K., Hart, J. K., Ong, R., "Environmental SensorNetworks," IEEE Computer, Vol. 37, No. 8, pp. 50-56.

4. Nikheel A. Chourasia, Surekha P. Washimkar," ZigBeeBased Wireless Air Pollution Monitoring" InternationalConference on Computing and Control Engineering(ICCCE 2012), 12 \& 13 April, 2012

5. R. Rajagopalan and P.K. Varshney, "DataAggregationTechniques in Sensor Networks: A Survey," IEEECommunication Surveys and Tutorials, Vol. 8 (4), pp.48-63, December 2006.

6. Mainwaring, A., Polastre, J., Szewczyk, R., Culler, D.,Anderson, J. "Wireless Sensor Networks for HabitatMonitoring," ACM International Workshop on WirelessSensor Networks and Applications, EUA.

\section{AUTHOR'S DETAILS}

Vijayakumar Sajjan, Research scholar, department of ECE from University Of Technology, Jaipur. He received B.E in 2011 from (BKEC) VTU, Belgaum, and M.tech in 2014 from (AIET) VTU, Belgaum. linkedin.com/in/vijay-sajjan-50759658 Vijay.sajjan26@gmail.com

Dr. Pramod Sharma, Professor In ECE Dept, University Of Technology, Jaipur. Scholastically advanced Educationist with rich scholarly and authoritative experience; offering a famous profession crossing more than 20 years in different Engineering Colleges. Achieve with sound reputation of inventive academic accomplishments. Master in arranging, sorting out, planning and checking a wide range of curricular and co-curricular exercises for the general advancement character of understudies and the board of the organizationlinkedin.com/in/dr-pramodsharma-9640a216 